\title{
The three-point correlation function of cosmic shear
}

\section{The natural components}

\author{
P. Schneider ${ }^{1,2}$ and M. Lombardi ${ }^{1}$ \\ 1 Institut für Astrophysik und Extraterrestrische Forschung, Universität Bonn, Auf dem Hügel 71, 53121 Bonn, Germany \\ e-mail: peter@astro.uni-bonn.de, lombardi@astro.uni-bonn.de \\ 2 Max-Planck-Institut für Astrophysik, Postfach 1317, 85741 Garching, Germany
}

Received 21 June 2002 / Accepted 18 October 2002

\begin{abstract}
The three-point correlation function of cosmic shear, the weak distortion of the images of distant galaxies by the gravitational field of the inhomogeneous matter distribution in the Universe, is studied here. Previous work on three-point statistics of cosmic shear has mainly concentrated on the convergence, or on aperture measures of the shear. However, as has become clear recently for the two-point statistics of cosmic shear, the basic quantity that should be used is the correlation function: first, it is much easier to measure from observational data, since it is immune against complicated geometries of data fields (which contain gaps and holes, e.g. due to masking); second, all other (linear) two-point statistics can be expressed as integrals over the correlation function. The situation is the same for the three-point statistics. However, in contrast to the twopoint correlation function, the invariants (with respect to rotations) of the shear three-point correlation function have not been employed yet. Here we consider the transformation properties of the shear three-point correlation function under rotations. We show that there are four complex linear combinations of components of the three-point correlation function, which we shall call "natural components", since they are multiplied just by a phase factor for arbitrary rotations, but do not mix. In particular, their moduli are invariant under rotations and thus (non-linear) invariants of the three-point correlation function. In terms of these natural components, the invariance of the statistical properties of the shear field under parity transformations are easily obtained. Our results do not apply only to cosmic shear, but also to other quantities with the same mathematical properties that of a polar. For example, practically every relation derived here applies also to the polarization of the cosmic microwave background radiation.
\end{abstract}

Key words. cosmology: theory - gravitational lensing - large-scale structure of the Universe - cosmic microwave background

\section{Introduction}

The weak gravitational lensing effect by the large-scale matter distribution of the Universe, called cosmic shear, has long been recognized as a unique tool to study the statistical properties of the cosmological (dark) matter distribution, without referring to luminous tracers of this distribution (Blandford et al. 1991; Miralda-Escude 1991; Kaiser 1992, 1998; Jain \& Seljak 1997; Bernardeau et al. 1997; Schneider et al. 1998; van Waerbeke et al. 1999; Bartelmann \& Schneider 1999; Jain et al. 2000; White \& Hu 2000; see Mellier 1999 and Bartelmann \& Schneider 2001 for recent reviews). Owing to the smallness of the effect, its actual measurement has only fairly recently been achieved, nearly simultaneously by several groups (Bacon et al. 2000; Kaiser et al. 2000; van Waerbeke et al. 2000; Wittman et al. 2000). This breakthrough became

Send offprint requests to: $\mathrm{P}$. Schneider,

e-mail: peter@astro.uni-bonn.de possible due to the usage of wide-field optical cameras and the development of special-purpose image analysis software specifically designed to measure the shape of very faint galaxies and to correct their ellipticity for effects of PSF smearing and anisotropy. By now, several additional cosmic shear measurements have been reported (Maoli et al. 2001; van Waerbeke et al. 2001; Rhodes et al. 2001; Bacon et al. 2002; Refregier et al. 2002; Hämmerle et al. 2002; Hoekstra et al. 2002), both from the ground and from HST imaging, partly with appreciably larger sky area than the original discovery papers.

In all of these papers, the cosmic shear signal detected was one related to the two-point correlation function of the shear, or some function of it, such as the shear dispersion or the aperture mass. To measure higher-order statistical properties of the cosmic shear, the quantity of data must be larger than for the second-order measures. It has been pointed out by a number of authors (e.g., Bernardeau et al. 1997; Jain \& Seljak 1997; Schneider et al. 1998; van Waerbeke et al. 1999; 
Hamana et al. 2002) that the third-order statistics (e.g. the skewness) contains very valuable cosmological information, such as the density parameter $\Omega_{\mathrm{m}}$. In particular, the neardegeneracy between $\sigma_{8}$ and $\Omega_{\mathrm{m}}$ in two-point cosmic shear statistics (see, e.g., van Waerbeke et al. 2002) can be broken if the three-point statistics is employed. Encouragingly, Bernardeau et al. (2002a) have reported the detection of a thirdorder statistical signal in their cosmic shear survey.

Apart from the larger difficulty to obtain a measurement of the third-order statistics of the cosmic shear, there is also the problem of an appropriate statistical estimator for the thirdorder shear. Whereas for the second-order, the statistically independent shear measures are known, we are not in this position for the third-order shear statistics. We shall briefly summarize the situation for the two-point statistics, and explain why the three-point shear statistics is substantially more complicated in Sect. 2 below. In Sect. 3 we shall then define the components of the shear three-point correlation function (3PCF) and study their transformation behavior under spatial rotations. From that, we shall then find in Sect. 4 the natural components of the shear 3PCF, which can be considered analogous to the natural components of the two-point correlation function of the shear. These components are "natural" in the sense that they have the simplest behavior under rotation transformations: each of the four complex natural components is just multiplied with a phase factor when an arbitrary rotation is applied, which in particular means that the moduli of these natural components are invariants under rotations. We shall discuss the importance of these natural components in Sect. 5, where we also outline the perspectives of future work that can be based on the use of these natural components. In an appendix, we shall consider the projection of the shear onto several particular reference points, defined by the various centers of a triangle.

We want to point out that all the relations derived in this papers are not confined only to cosmic shear. In fact, this paper investigates the three-point correlation function of a polar - a polar is a two-component quantity which transforms under a rotation of the coordinate frame by a phase factor $\mathrm{e}^{2 \mathrm{i} \varphi}$. Alternatively, a polar can be viewed as the trace-free part of a symmetric $2 \times 2$ matrix. In the case of cosmic shear, this matrix is the Hessian of the deflection potential. Another polar of great cosmological importance is the polarization of the cosmic microwave background. Hence, all the results presented below do equally well apply to the three-point correlation function of the CMB polarization.

\section{Motivation}

For the two-point cosmic shear statistics, the basic quantities are the two-point correlation functions. Given a pair of points, $\boldsymbol{X}_{i}$, and the Cartesian components of the shear $\gamma_{\mu}\left(\boldsymbol{X}_{i}\right)$ there ( $i=1,2, \mu=1,2$ ), one projects the shear along the direction $\varphi$ connecting these two points by defining the tangential and cross component, $\gamma_{\mathrm{t}}$ and $\gamma_{\times}$by $\gamma_{\mathrm{t} i}+\mathrm{i} \gamma_{\times i}=-\left[\gamma_{1}\left(\boldsymbol{X}_{i}\right)+\mathrm{i} \gamma_{2}\left(\boldsymbol{X}_{i}\right)\right] \mathrm{e}^{-2 \mathrm{i} \varphi}$. Then, one forms the correlation functions $\xi_{\mathrm{tt}}(\theta)=\left\langle\gamma_{\mathrm{t} 1} \gamma_{\mathrm{t} 2}\right\rangle$ and $\xi_{\times \times}(\theta)=\left\langle\gamma_{\times 1} \gamma_{\times 2}\right\rangle$, where the average is an ensemble average over all pairs of points with separation $\theta$. Even more useful are the linear combinations $\xi_{ \pm}(\theta)=\xi_{\mathrm{tt}}(\theta) \pm \xi_{\times \times}(\theta)$. Another combination which one may be tempted to take is $\left\langle\gamma_{\mathrm{t} 1} \gamma_{\times 2}\right\rangle$, but this changes sign under parity transformations and thus should vanish. All other two-point statistical measures of the cosmic shear, such as the shear dispersion in a circle or the aperture mass dispersion, can be expressed as integrals over these two correlation functions (e.g., Crittenden et al. 2002; Schneider et al. 2002a). From a practical point of view, the determination of the shear correlation functions is also most convenient, as they can be measured also in data fields of complicated geometry (as normally data fields are, due to masking). The two correlation functions $\xi_{ \pm}$can be expressed readily in terms of the power spectrum of the mass distribution in the Universe.

Compared with this situation, a proper measure of the three-point shear statistics is much more difficult to define. First we note that the two-point function $\xi_{+}=\left\langle\gamma \gamma^{*}\right\rangle$ (where "*" denotes complex conjugation) can be defined without any reference direction; this is not the case for any three-point function of the shear, since with three two-component quantities alone, no tri-linear scalar can be formed. Hence, one needs to project the shear components. In contrast to the case of two points, where there is a unique choice of the reference direction, this is no longer true for a triangle: there is not a single "natural direction" defined in a triangle; in fact, there are several of those (see the appendix). Therefore, it is not a priory clear how to define "useful" components of the three-point correlation function. One might ask, for example, whether there are similar "invariant" combinations of the components of the shear 3PCF as there are for the two-point function $\left(\xi_{ \pm}\right)$.

Given these difficulties, it is not surprising that the work on the three-point statistics of cosmic shear has been relatively sparse. Bernardeau et al. (1997) and van Waerbeke et al. (1999) consider the 3PCF of the surface mass density as reconstructed from the shear measurements. Whereas possible in principle, the fact that real data sets have gaps and holes makes the reconstructed mass map susceptible to systematics due to the geometry. Schneider et al. (1998) suggested to use the aperture mass (Schneider 1996) as a cosmic shear statistics for which the third-order moment is readily calculated directly from the shear data. However, as is the case for the shear dispersion, one needs to cover the data field with (circular) apertures which presents again a problem in case of gaps in the data. Recognizing this, Bernardeau et al. (2002b) defined a particular component (or, more precisely, a particular linear combination of components) of the 3PCF that is readily measured from observational data, calculated its expectation value from numerical ray-tracing simulations of Jain et al. (2000) and successfully applied it to the VIRMOS-DESCART survey in Bernardeau et al. (2002a).

In addition to the component of the three-point shear correlator considered by Bernardeau (2002b), the other components (there are a total of 8) may contain equally valuable information about the bispectrum of the mass distribution. In addition, it is easily seen that all measures of the third-order shear statistics can be expressed as integrals over the shear 3PCF. They are easiest to determine from real data and shall therefore be considered as the basic quantities. In this paper we will derive the "natural" components of the shear 3PCF, by considering their transformation behavior under rotations. 


\section{The shear three-point correlation function, and the centers of a triangle}

\subsection{Definition of the 3PCF}

Consider three points $\boldsymbol{X}_{l}, 1 \leq l \leq 3$, and define their difference vectors $\boldsymbol{x}_{1}=\boldsymbol{X}_{3}-\boldsymbol{X}_{2}, \boldsymbol{x}_{2}=\boldsymbol{X}_{1}-\boldsymbol{X}_{3}, \boldsymbol{x}_{3}=\boldsymbol{X}_{2}-\boldsymbol{X}_{1}$, so that $\boldsymbol{x}_{1}+\boldsymbol{x}_{2}+\boldsymbol{x}_{3}=\mathbf{0}$ (see Fig. 1). Each of the three difference vectors will be written as $\boldsymbol{x}_{l}=\left(x_{l} \cos \varphi_{l}, x_{l} \sin \varphi_{l}\right)$, so that $\varphi_{l}$ is the orientation of the $l$ th side of the triangle. Furthermore, we define $\phi_{l}$ as being the interior angle of the triangle at the corner $\boldsymbol{X}_{l}$. In order to have general relations, in this paper we will use oriented angles, i.e. we will attach to each angle a sign indicating its orientation. More precisely, we will define $\phi_{1} \in(-\pi,+\pi)$ to have the same sign of the cross product $\boldsymbol{x}_{2} \times \boldsymbol{x}_{3}$ (where $\boldsymbol{a} \times \boldsymbol{b}:=a_{1} b_{2}-a_{2} b_{1}$ ), and similarly for $\phi_{2}$ and $\phi_{3}$. Note that, since $\boldsymbol{x}_{1} \times \boldsymbol{x}_{2}=\boldsymbol{x}_{2} \times \boldsymbol{x}_{3}=\boldsymbol{x}_{3} \times \boldsymbol{x}_{1}$ (which follows from the vanishing of the sum of the $x_{l}$, or in a more geometric way, from the fact that each of these cross products equals twice the area of the triangle), all angles $\phi_{l}$ will be either positive or negative; in particular, they all will be positive if the closed path from $X_{1}$ to $X_{2}$ to $X_{3}$ to $X_{1}$ goes around the triangle counterclockwise. We also observe that this convention for the angles implies that the sum of the internal angles of the triangle will be $\pm \pi$ depending on the orientation; however, this ambiguity will not generally play a role, since all relations for angles are defined modulo $2 \pi$. In the following we will call a triangle positively (respectively, negatively) oriented if the sum of its internal angle is $+\pi(-\pi)$. The relation between the $\phi_{l}$ and the $\varphi_{l}$ is given by

$\varphi_{3}-\varphi_{2}=\pi-\phi_{1} ; \quad \varphi_{1}-\varphi_{3}=\pi-\phi_{2} ; \quad \varphi_{2}-\varphi_{1}=\pi-\phi_{3}$.

Let $\gamma_{\mu}\left(\boldsymbol{X}_{l}\right)$ be the Cartesian components of the shear at point $\boldsymbol{X}_{l}$; they are defined in terms of the deflection potential $\psi(\boldsymbol{X})$ by the differential operation $\gamma_{1}=\left[\partial^{2} \psi /\left(\partial X_{1}^{2}\right)-\partial^{2} \psi /\left(\partial X_{2}^{2}\right)\right] / 2$, $\gamma_{2}=\partial^{2} \psi /\left(\partial X_{1} \partial X_{2}\right)$. We define the Cartesian components of the shear $3 \mathrm{PCF}$ as

$\gamma_{\mu \nu \lambda}\left(\boldsymbol{x}_{1}, \boldsymbol{x}_{2}, \boldsymbol{x}_{3}\right) \equiv\left\langle\gamma_{\mu}\left(\boldsymbol{X}_{1}\right) \gamma_{\nu}\left(\boldsymbol{X}_{2}\right) \gamma_{\lambda}\left(\boldsymbol{X}_{3}\right)\right\rangle$,

where we have made use of the fact that the shear field is assumed to be a homogeneous random field, so that the 3PCF is invariant under translations; therefore, $\gamma_{\mu \nu \lambda}$ does depend only on the separation vectors $\boldsymbol{x}_{l}$. Although we also assume that the random field is isotropic, the Cartesian components $\gamma_{\mu \nu \lambda}$ of the 3PCF do not depend just on the $x_{l}=\left|\boldsymbol{x}_{l}\right|$ (see below).

For any reference direction $\zeta_{l}$, we can define the tangential and cross components of the shear, $\gamma_{l t}$ and $\gamma_{l \times}$, respectively, at point $\boldsymbol{X}_{l}$ relative to this direction by

$$
\begin{aligned}
\gamma\left(\boldsymbol{X}_{l}, \zeta_{l}\right) & \equiv \gamma_{\mathrm{t}}\left(\boldsymbol{X}_{l}, \zeta_{l}\right)+\mathrm{i} \gamma_{\times}\left(\boldsymbol{X}_{l}, \zeta_{l}\right): \\
& =-\gamma\left(\boldsymbol{X}_{l}\right) \mathrm{e}^{-2 \mathrm{i} \zeta_{l}}=-\left[\gamma_{1}\left(\boldsymbol{X}_{l}\right)+\mathrm{i} \gamma_{2}\left(\boldsymbol{X}_{l}\right)\right] \mathrm{e}^{-2 \mathrm{i} \zeta_{l}},
\end{aligned}
$$

or explicitly in terms of the components,

$\gamma_{\mu}\left(\boldsymbol{X}_{l}, \zeta_{l}\right)=-R_{\mu v}\left(2 \zeta_{l}\right) \gamma_{v}\left(\boldsymbol{X}_{l}\right)$

where $R_{\mu \nu}(\varphi)=\delta_{\mu \nu} \cos \varphi+\epsilon_{\mu \nu} \sin \varphi$ is the rotation matrix, $\delta_{\mu v}$ the Kronecker delta, and $\epsilon_{12}=1=-\epsilon_{21}, \epsilon_{11}=0=\epsilon_{22}$; here and in the following, we identify the tangential component

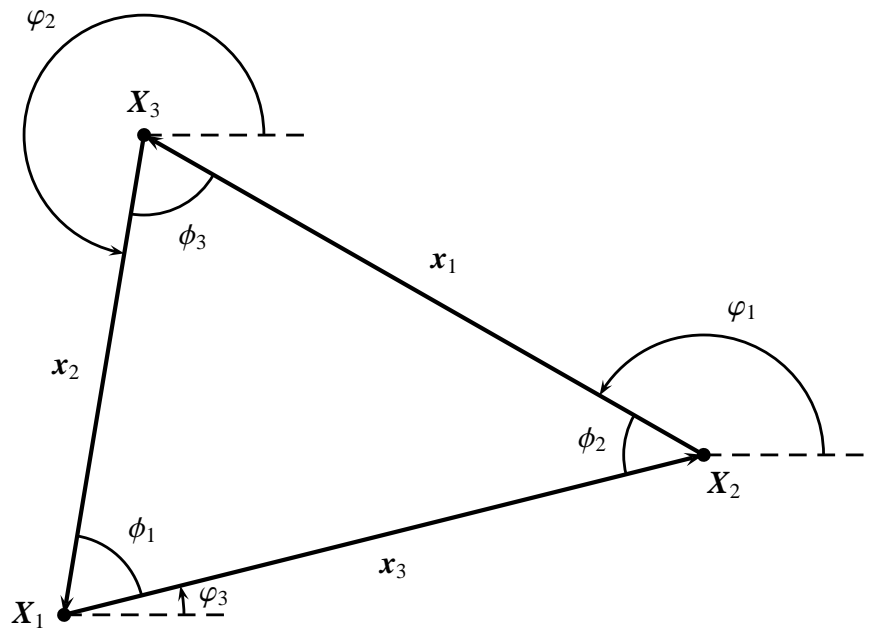

Fig. 1. Definitions of the geometry of a triangle. The $\varphi_{l}$ are the orientations of the sides of the triangle relative to the positive $x_{1}$-direction, the $\phi_{l}$ are the interior angles of the triangle, which are related to the $\varphi_{l}$ by (1), provided the orientation of the three points is as displayed here.

with $\mu=1$, and the cross component with $\mu=2$ when we write transformation equations like (4), and we employ the Einstein summation convention. We notice that, because of the rotation behavior of the shear, we only need to specify the projection direction $\zeta_{l}$ modulo $\pi$.

If the reference directions $\zeta_{l}$ are defined in terms of the position vectors of the vertices of the triangle, or the side vectors, and thus rotate in the same way as the triangle as whole, then the tangential and cross components of the shear are invariant under rotations and translations of the triangle. For example, the direction $\zeta_{l}$ could be chosen as the direction $\varphi_{l}$ of the opposite side of the triangle. The 3PCF of these projected shear components will then depend only on the $\left|\boldsymbol{x}_{l}\right|$,

$$
\begin{aligned}
\gamma_{\mu \nu \lambda}^{\left(\zeta_{l}\right)}\left(x_{1}, x_{2}, x_{3}\right) & = \\
& -R_{\mu \alpha}\left(2 \zeta_{1}\right) R_{\nu \beta}\left(2 \zeta_{2}\right) R_{\lambda \gamma}\left(2 \zeta_{3}\right) \gamma_{\alpha \beta \gamma}\left(\boldsymbol{x}_{1}, \boldsymbol{x}_{2}, \boldsymbol{x}_{3}\right)
\end{aligned}
$$

The orientation of the triangle becomes important if the arguments of the 3PCF are written as the three side lengths; in this case, the triangle is defined only up to a parity transformation. Hence, in all relations involving only the side lengths of a triangle, we will assume that the triangle has positive parity. We shall see in Sect. 4 how the shear 3PCF behaves under parity transformations.

In Eq. (5), the directions $\zeta_{l}$ are arbitrary. For a given set of three points, i.e., for a given triangle, there are several natural choices for the reference directions; we shall discuss those in the next subsection. One choice was already mentioned above, namely the direction $\varphi_{l}$ of the side opposite to the corner $\boldsymbol{X}_{l}$. We shall label the corresponding 3PCF with the superscript "s" (for "side"),

$$
\begin{aligned}
& \gamma_{\mu \nu \lambda}^{(\mathrm{s})}\left(x_{1}, x_{2}, x_{3}\right)= \\
& \quad-R_{\mu \alpha}\left(2 \varphi_{1}\right) R_{\nu \beta}\left(2 \varphi_{2}\right) R_{\lambda \gamma}\left(2 \varphi_{3}\right) \gamma_{\alpha \beta \gamma}\left(x_{1}, x_{2}, x_{3}\right) .
\end{aligned}
$$




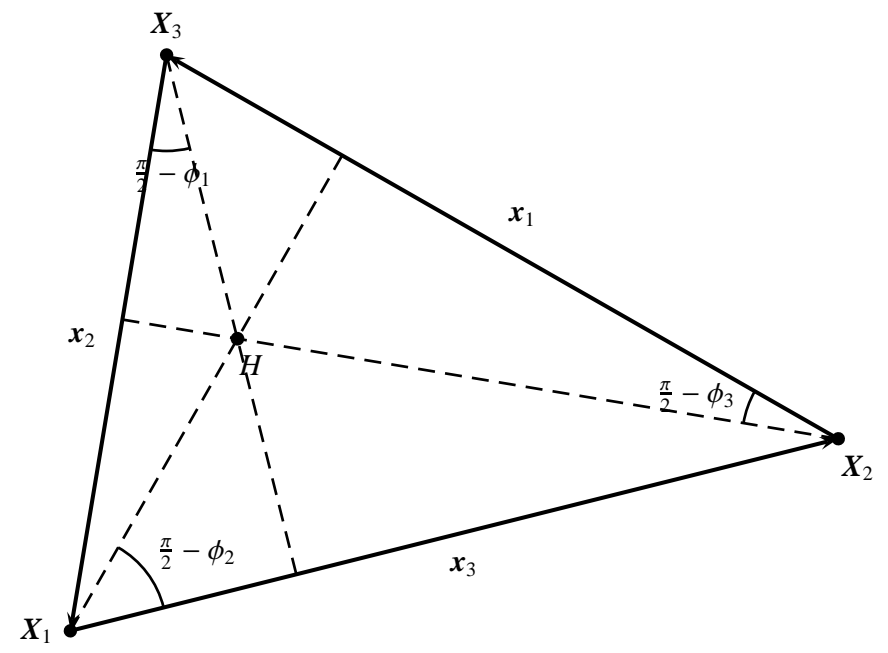

Fig. 2. The orthocenter of a triangle, determined by the intersection of the three altitudes. The angle between the altitude of $\boldsymbol{x}_{1}$ and the side $\boldsymbol{x}_{3}$ is easily obtained from the right triangle formed by this altitude and the two sides $\boldsymbol{x}_{1}$ and $\boldsymbol{x}_{3}$.

\subsection{The centers of a triangle, and the corresponding shear projections}

In addition to the side projection mentioned above, there are several other natural choices for the directions along which the shear can be projected. For each triangle, one can define a number of "centers"; the direction of the vector connecting the point $\boldsymbol{X}_{l}$ with one of these centers can be used to define convenient components of the 3PCF. The four most important centers are: (1) the centroid of a triangle; it is the point where the side-bisectors intersect; (2) the incenter (center of the incircle), which is the intersection of the three angle-bisectors of the interior angles $\phi_{l}$; (3) the circumcenter (center of the circumcircle), which is the point of intersection of the three midperpendiculars, and (4) the orthocenter, which is the intersection point of the altitudes.

We now define the tangential and cross components of the shear relative to the direction of the line connecting the point $\boldsymbol{X}_{l}$ with one of these centers. Since the line connecting $\boldsymbol{X}_{l}$ with the orthocenter (the point " $H$ " in Fig. 2) is perpendicular to the side vector $\boldsymbol{x}_{l}$, we find for the projection of the shear relative to the direction of the orthocenter (labeled with superscript "o") simply by setting $\zeta_{l}=\varphi_{l}+\pi / 2$ in (4),

$\gamma_{\mu}^{(\mathrm{o})}=-\gamma_{\mu}^{(\mathrm{s})}$.

Therefore, we obtain the shear $3 \mathrm{PCF}$ for this projection from Eq. (5)

$\gamma_{\mu \nu \lambda}^{(\mathrm{o})}\left(x_{1}, x_{2}, x_{3}\right)=-\gamma_{\mu \nu \lambda}^{(\mathrm{s})}\left(x_{1}, x_{2}, x_{3}\right)$.

Next we consider the center of the incircle. As this is given by the intersection of the angle-bisectors, we obtain for the direction $\zeta_{1}$ of the line connecting $\boldsymbol{X}_{1}$ with the incenter (see Fig. 3) $\zeta_{1}=\varphi_{3}+\phi_{1} / 2 ;$ hence,

$\gamma_{\mu}^{(\mathrm{in})}\left(\boldsymbol{X}_{1}\right)=-R_{\mu v}\left(2 \varphi_{3}+\phi_{1}\right) \gamma_{v}\left(\boldsymbol{X}_{1}\right)$

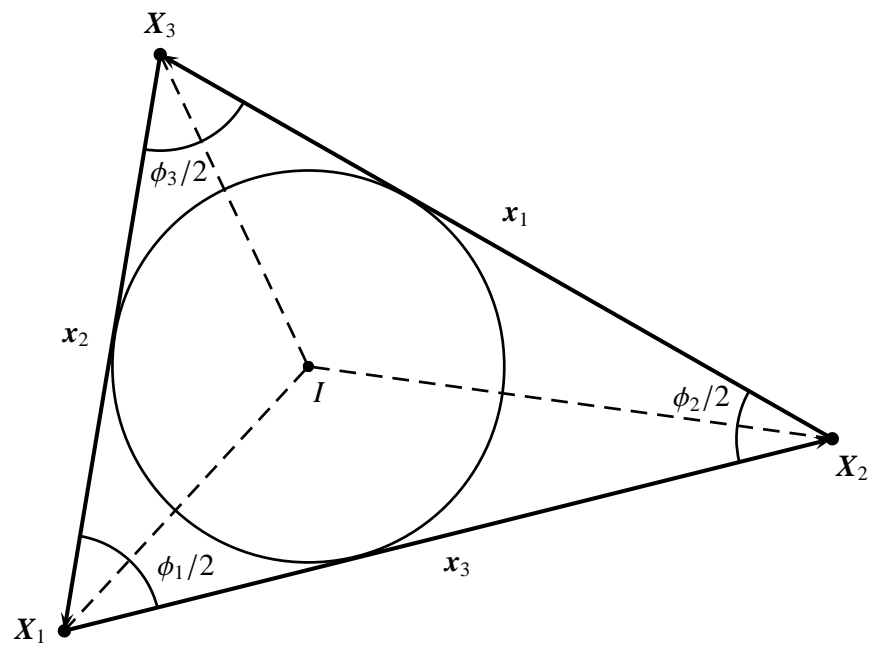

Fig. 3. The incenter of a triangle is given by the intersection of the three interior angle-bisectors.

Using Eqs. (4) and (7), one finds that the relation between the Cartesian components of the shear and those defined with respect to the ortho-center is

$\gamma_{\mu}\left(\boldsymbol{X}_{l}\right)=R_{\mu \nu}\left(-2 \varphi_{l}\right) \gamma_{v}^{(\mathrm{o})}\left(\boldsymbol{X}_{l}\right)$,

so that

$\gamma_{\mu}^{(\mathrm{in})}\left(\boldsymbol{X}_{1}\right)=-R_{\mu v}\left(2 \varphi_{3}-2 \varphi_{1}+\phi_{1}\right) \gamma_{v}^{(\mathrm{o})}\left(\boldsymbol{X}_{1}\right)$.

Since $2 \varphi_{3}-2 \varphi_{1}+\phi_{1}=-2 \pi+2 \phi_{2}+\phi_{1}=-\pi+\phi_{2}-\phi_{3}$, where we used Eq. (1) and the fact that the sum of the $\phi_{l}$ is $\pi$, one finally obtains

$\gamma_{\mu \nu \lambda}^{(\mathrm{in})}=R_{\mu \alpha}\left(\phi_{2}-\phi_{3}\right) R_{\nu \beta}\left(\phi_{3}-\phi_{1}\right) R_{\lambda \gamma}\left(\phi_{1}-\phi_{2}\right) \gamma_{\alpha \beta \gamma}^{(\mathrm{o})}$,

where the result for the other two points were obtained from the one at $\boldsymbol{X}_{1}$ by cyclic permutation of the indices. It should be noted that in this equation, as in all the following, the angles $\phi_{l}$ are functions of the sidelengths $x_{l}$ (and the orientation), and not independent geometrical quantities; hence, an equation like (10) contains only the $x_{l}$ as independent variables.

Next we consider the projection onto the center of the circumcircle. If $\zeta_{1}$ denotes the direction of the line connecting $\boldsymbol{X}_{1}$ with this center, then the shear components with respect to this center (denoted by the superscript "out") read

$\gamma_{\mu}^{(\text {out })}=-R_{\mu \nu}\left(2 \zeta_{1}\right) \gamma_{\nu}\left(X_{1}\right)=-R_{\mu \nu}\left(2 \zeta_{1}-2 \varphi_{1}\right) \gamma_{\nu}^{(\mathrm{o})}\left(\boldsymbol{X}_{1}\right)$,

where in the second step we used Eq. (9). From Thales' theorem (see Fig. 5) one finds that $\zeta_{1}-\varphi_{3}= \pm \pi / 2-\phi_{3}$, where the sign \pm depends on the orientation of the triangle. Hence, $\zeta_{1}-\varphi_{1}= \pm \pi / 2-\phi_{3}+\varphi_{3}-\varphi_{1}=\mp \pi / 2+\phi_{2}-\phi_{3}$, and we see that the sign ambiguity does not play a role because of the rotation properties of the shear (cf. comment after Eq. (4)). For the other two points, the corresponding relations are obtained by cyclic permutations, so that

$\gamma_{\mu \nu \lambda}^{\text {(out) }}=R_{\mu \alpha}\left(2 \phi_{2}-2 \phi_{3}\right) R_{\nu \beta}\left(2 \phi_{3}-2 \phi_{1}\right) R_{\lambda \gamma}\left(2 \phi_{1}-2 \phi_{2}\right) \gamma_{\alpha \beta \gamma}^{(\mathrm{o})}$.

Finally, we consider the projection on the center of mass of the triangle, or the centroid (denoted by the superscript "cen"), 


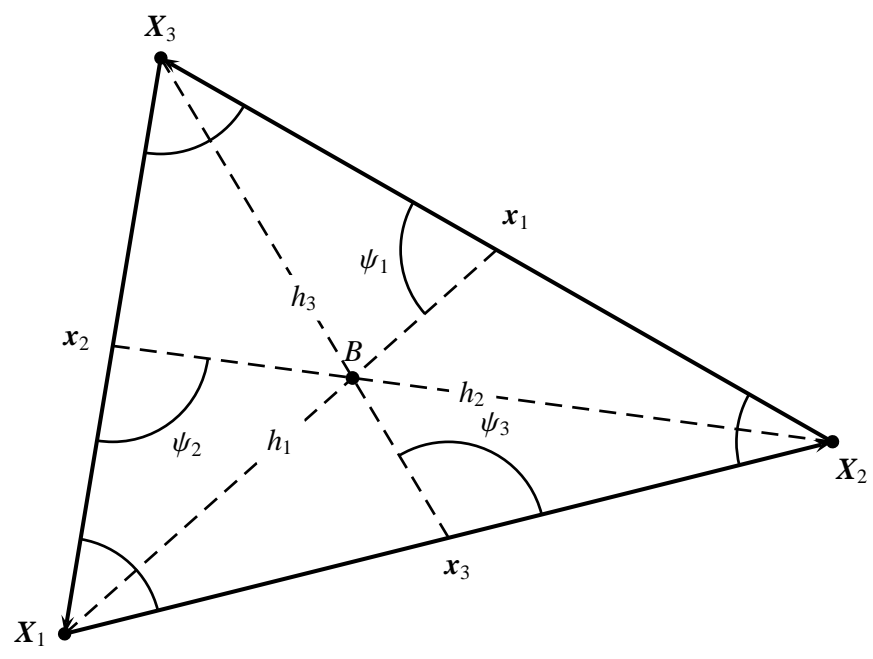

Fig. 4. The centroid of a triangle is the intersection point of the three side-bisectors.

which is the intersection of the three side-bisectors. Denoting by $\psi_{3}$ the angle between the side bisector of side $x_{3}$ and $\boldsymbol{x}_{3}$, we obtain from Fig. 4 that

$\sin \psi_{3}=\frac{x_{1}}{h_{3}} \sin \phi_{2}=\frac{x_{1} x_{2}}{h_{3} x_{3}} \sin \phi_{3}$,

where we made use of the sine-theorem, and

$h_{3}=\frac{1}{2} \sqrt{2 x_{1}^{2}+2 x_{2}^{2}-x_{3}^{2}}$

is the length of the side-bisector of side $x_{3}$. From the same figure we also obtain that

$\cos \psi_{3}=\frac{h_{3}^{2}+x_{3}^{2} / 4-x_{1}^{2}}{h_{3} x_{3}}=\frac{x_{2}^{2}-x_{1}^{2}}{2 h_{3} x_{3}}$,

so that

$\cos 2 \psi_{3}=\frac{\left(x_{2}^{2}-x_{1}^{2}\right)^{2}-4 x_{1}^{2} x_{2}^{2} \sin ^{2} \phi_{3}}{4 h_{3}^{2} x_{3}^{2}} ;$

$\sin 2 \psi_{3}=\frac{\left(x_{2}^{2}-x_{1}^{2}\right) x_{1} x_{2} \sin \phi_{3}}{h_{3}^{2} x_{3}^{2}}$.

Analogous relations are obtained for the other two points by cyclic permutations of the indices. The shear components projected toward the centroid are then

$\gamma_{\mu}^{(\mathrm{cen})}\left(\boldsymbol{X}_{l}\right)=R_{\mu v}\left(2 \psi_{l}\right) \gamma^{(\mathrm{s})}=-R_{\mu v}\left(2 \psi_{l}\right) \gamma^{(\mathrm{o})}$,

where the components of the rotation matrix can be directly obtained from Eq. (13). For completeness, we give the 3PCF for the centroid,

$\gamma_{\mu \nu \lambda}^{(\mathrm{cen})}=-R_{\mu \alpha}\left(2 \psi_{1}\right) R_{\nu \beta}\left(2 \psi_{2}\right) R_{\lambda \gamma}\left(2 \psi_{3}\right) \gamma_{\alpha \beta \gamma}^{(\mathrm{o})}$.

\section{Invariant components of the shear three-point correlation function}

The normal way to choose "good" components of a multi-index quantity like $\gamma_{\mu v \lambda}$ is to look for its behavior under coordinate

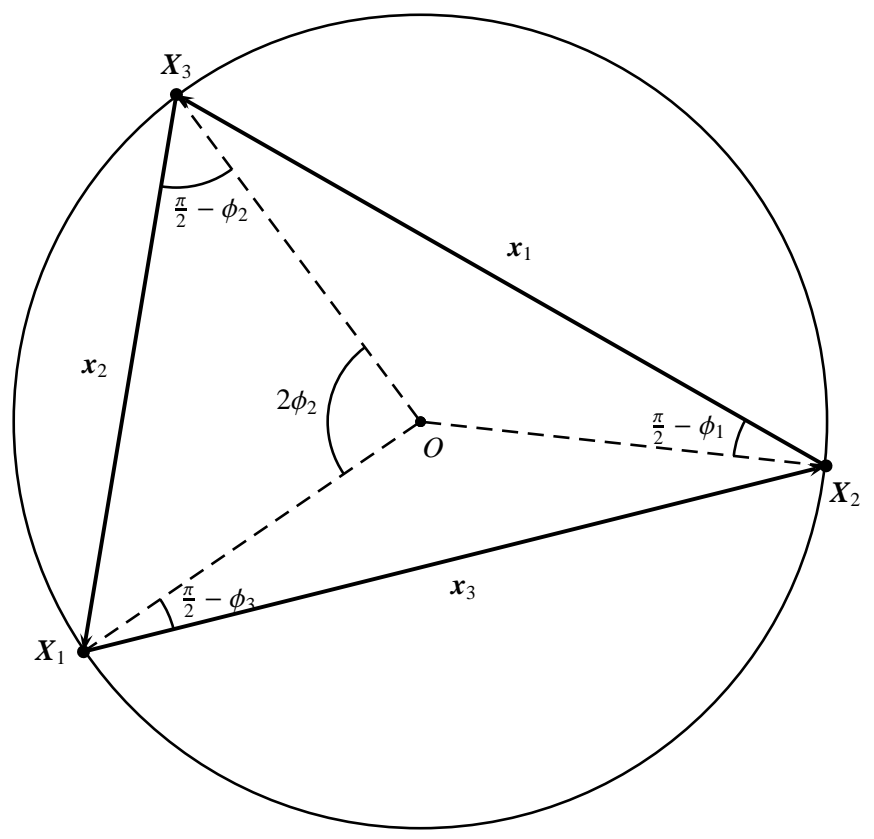

Fig. 5. The circumcenter of a triangle is given by the intersection of the three midperpendiculars. According to Thales' theorem, the side $x_{2}$ subtends the angle $2 \phi_{2}$ as seen from the circumcenter; from that, the angle between the lines connecting the points $\boldsymbol{X}_{l}$ with the circumcenter are easily obtained by noting that all three of them have equal length.

transformations. Recall the situation for the shear two-point correlation function: the correlator $\left\langle\gamma_{\mu} \gamma_{\nu}\right\rangle(\boldsymbol{x})$ of the Cartesian components of the shear contains two terms, one which is independent on the phase $\varphi$ of $\boldsymbol{x}$, the other behaving as $\cos (4 \varphi)$. The coefficients of these two terms are $\xi_{+}$and $\xi_{-}$, respectively, which are the invariants, and therefore the natural choice for the components of the two-point correlation function of the shear.

Similarly, we can consider the behavior of the 3PCF under rotations. Given that no linear scalar can be built from the Cartesian components of the 3PCF alone we cannot expect to find a combination of components which is invariant under general rotations. However, as we shall see, there are linear combinations of the components of the 3PCF which have a simple behavior under rotations, namely multiplication by a phase factor. Owing to this simple transformation behavior, we shall term them "natural" components.

Let now $\gamma_{\mu \nu \lambda}$ be the components of the 3PCF of the shear measured with respect to one particular choice of direction(s); choosing different projection directions, which differ from the old ones by $\zeta_{i}$, the 3PCF becomes

$$
\begin{aligned}
\gamma_{\mu \nu \lambda}^{\prime} & \equiv\left\langle\gamma_{\mu}^{\prime}\left(\boldsymbol{X}_{1}\right) \gamma_{\nu}^{\prime}\left(\boldsymbol{X}_{2}\right) \gamma_{\lambda}^{\prime}\left(\boldsymbol{X}_{3}\right)\right\rangle \\
& =R_{\mu \alpha}\left(2 \zeta_{1}\right) R_{\nu \beta}\left(2 \zeta_{2}\right) R_{\lambda \gamma}\left(2 \zeta_{3}\right) \gamma_{\alpha \beta \gamma}
\end{aligned}
$$

note that this expression differs from Eq. (5) by a minus sign since in Eq. (5) the transition from Cartesian to tangential and cross components was included as well. To investigate more closely the action of the rotation operator in Eq. (16), we transform this equation into one which appears more familiar: We define the eight-component quantity

$\Gamma:=\left(\gamma_{\mathrm{ttt}}, \gamma_{\mathrm{tt} \times}, \gamma_{\mathrm{t} \times \mathrm{t}}, \gamma_{\mathrm{t} \times \times}, \gamma_{\times \mathrm{tt}}, \gamma_{\times \mathrm{t} \times}, \gamma_{\times \times \mathrm{t}}, \gamma_{\times \times \times}\right)$ 
and analogously $\Gamma^{\prime}$ for the transformed components; then, the rotation described in Eq. (16) can be written as $\Gamma^{\prime}=R \Gamma$, where $R$ is a $8 \times 8$ matrix. The components of $R$ are triple products of trigonometric functions. Since it describes a rotation, $R$ is unitary and one expects that the eigenvalues of $R$ have an absolute value of unity. They can in fact be obtained as

$\lambda_{1,2}^{(0)}=\exp \left( \pm 2 \mathrm{i}\left[\zeta_{1}+\zeta_{2}+\zeta_{3}\right]\right) ; \lambda_{1,2}^{(1)}=\exp \left( \pm 2 \mathrm{i}\left[-\zeta_{1}+\zeta_{2}+\zeta_{3}\right]\right) ;$

$\lambda_{1,2}^{(2)}=\exp \left( \pm 2 \mathrm{i}\left[\zeta_{1}-\zeta_{2}+\zeta_{3}\right]\right) ; \quad \lambda_{1,2}^{(3)}=\exp \left( \pm 2 \mathrm{i}\left[\zeta_{1}+\zeta_{2}-\zeta_{3}\right]\right)$.

Note that this result is not very surprising: either the eigenvalues are \pm 1 , or they have to appear in the above form, i.e., they have to occur as pairs of complex conjugate numbers with absolute value of unity. The dependence of the eigenvalues on the rotation angles is in fact a natural one.

\subsection{The natural components of the 3PCF}

We see that under the general rotation described by Eq. (16), the eigenvalue +1 does not occur; in other words, there is no (linear) combination of the components of the 3PCF that is invariant under the transformation (16). However, for some special rotations, the eigenvalue +1 occurs. As a first example, we consider the case $\zeta_{1}+\zeta_{2}+\zeta_{3}=0$; as shown in Sect. 3.2, this case actually is encountered for transformations of the shear components between the orthocenter, the incenter and the circumcenter. Then, the two eigenvalues $\lambda_{1,2}^{(0)}$ are +1 , and the corresponding eigenvectors can be found to be

$E_{1}^{(0)}=(1,0,0,-1,0,-1,-1,0)$;

$E_{2}^{(0)}=(0,1,1,0,1,0,0,-1)$.

Hence, under rotations of this kind, we expect that the two combinations

$\Gamma_{1}^{(0)}=\gamma_{\mathrm{ttt}}-\gamma_{\mathrm{t} \times \mathrm{x}}-\gamma_{\times \mathrm{t} \times}-\gamma_{\times \times \mathrm{t}} ; \quad \Gamma_{2}^{(0)}=\gamma_{\mathrm{tt} \times}+\gamma_{\mathrm{t} \times \mathrm{t}}+\gamma_{\times \mathrm{tt}}-\gamma_{\times \times \times}$

of the components of the $3 \mathrm{PCF}$ are both invariant. It will turn out to be very useful to combine these two invariants into a single complex quantity, $\Gamma^{(0)}=\Gamma_{1}^{(0)}+\mathrm{i} \Gamma_{2}^{(0)}$. This quantity, however, can also be written in a different form, namely

$\Gamma^{(0)}=\left\langle\gamma\left(\boldsymbol{X}_{1}\right) \gamma\left(\boldsymbol{X}_{2}\right) \gamma\left(\boldsymbol{X}_{3}\right)\right\rangle$,

where we again consider $\gamma=\gamma_{\mathrm{t}}+\mathrm{i} \gamma_{\times}$as a complex quantity. Written in this form, it is obvious that a rotation with $\zeta_{1}+\zeta_{2}+$ $\zeta_{3}=0$ keeps $\Gamma^{(0)}$ invariant.

Next we consider the case that $-\zeta_{1}+\zeta_{2}+\zeta_{3}=0$. Such a rotation occurs if one transforms the shear components from the incenter (or the out- or orthocenter) to the center of one of the escribed circles (see Fig. 6 for an explanation); in this case, $\lambda_{1,2}^{(1)}=1$, and from the corresponding eigenvectors one can again construct two combinations $\Gamma_{1}^{(1)}, \Gamma_{2}^{(1)}$, of the components of the $3 \mathrm{PCF}$ that stay invariant under such a rotation, and they can as well be combined into a single complex quantity. The same procedure can then be repeated for rotations with $\zeta_{1}-\zeta_{2}+\zeta_{3}=0$ and those with $\zeta_{1}+\zeta_{2}-\zeta_{3}=0$; these correspond, e.g., to the transformation from one of the aforementioned centers to the centers of the other two escribed circles.

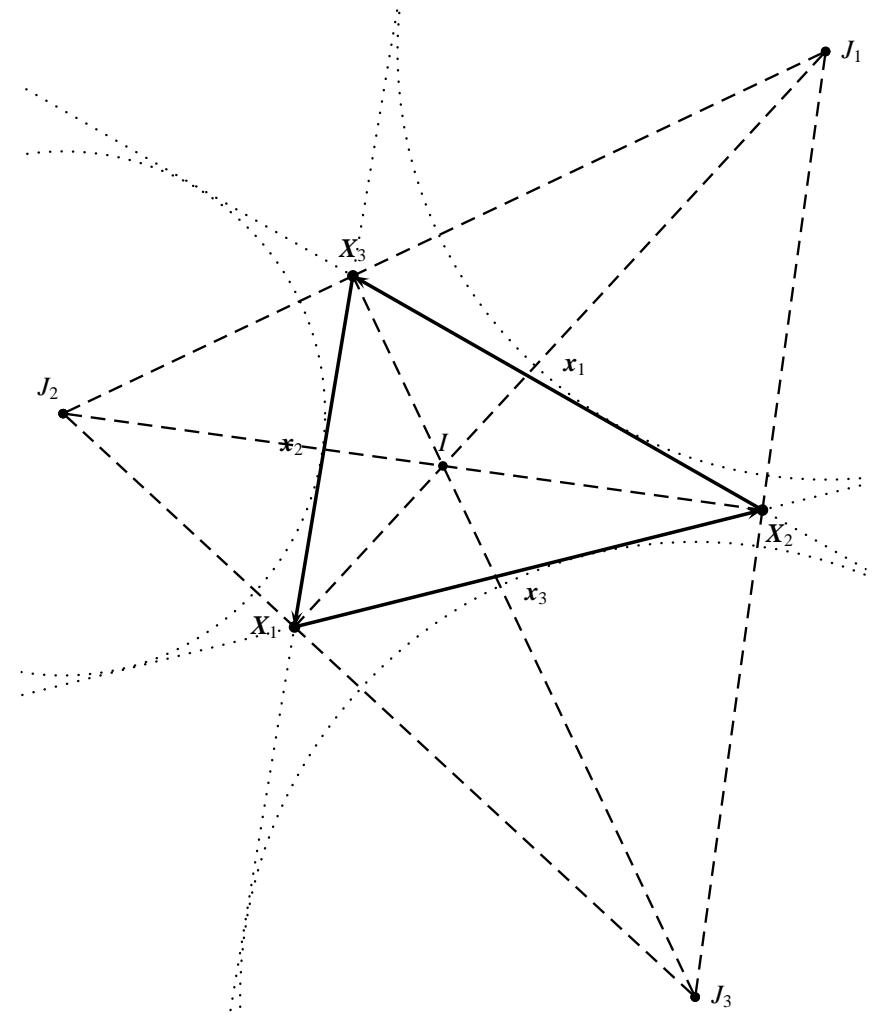

Fig. 6. The centers of the escribed circles are intersections between one interior angle bisector and two exterior angle bisectors. These three escribed circles are tangent to one side of the triangle and the extensions of the two other sides.

The corresponding invariants read:

$$
\begin{aligned}
\Gamma^{(1)} & =\gamma_{\mathrm{ttt}}-\gamma_{\mathrm{t} \times \times}+\gamma_{\times \mathrm{tx}}+\gamma_{\times \times \mathrm{t}}+\mathrm{i}\left[\gamma_{\mathrm{tt} \times}+\gamma_{\mathrm{t \times t}}-\gamma_{\times \mathrm{tt}}+\gamma_{\times \times \times}\right] \\
& =\left\langle\gamma^{*}\left(\boldsymbol{X}_{1}\right) \gamma\left(\boldsymbol{X}_{2}\right) \gamma\left(\boldsymbol{X}_{3}\right)\right\rangle ; \\
\Gamma^{(2)} & =\gamma_{\mathrm{ttt}}+\gamma_{\mathrm{t} \times \times}-\gamma_{\times \mathrm{tx}}+\gamma_{\times \times \mathrm{t}}+\mathrm{i}\left[\gamma_{\mathrm{tt} \times}-\gamma_{\mathrm{t} \times \mathrm{t}}+\gamma_{\times \mathrm{tt}}+\gamma_{\times \times \times}\right] \\
& =\left\langle\gamma\left(\boldsymbol{X}_{1}\right) \gamma^{*}\left(\boldsymbol{X}_{2}\right) \gamma\left(\boldsymbol{X}_{3}\right)\right\rangle ; \\
\Gamma^{(3)} & =\gamma_{\mathrm{ttt}}+\gamma_{\mathrm{t} \times \times}+\gamma_{\times \mathrm{tx} \times}-\gamma_{\times \times \mathrm{t}}+\mathrm{i}\left[-\gamma_{\mathrm{tt} \times}+\gamma_{\mathrm{t} \times \mathrm{t}}+\gamma_{\times \mathrm{tt}}+\gamma_{\times \times \times}\right] \\
& =\left\langle\gamma\left(\boldsymbol{X}_{1}\right) \gamma\left(\boldsymbol{X}_{2}\right) \gamma^{*}\left(\boldsymbol{X}_{3}\right)\right\rangle
\end{aligned}
$$

Now, each of these $\Gamma^{(\alpha)}$ is invariant only under special rotations, as the derivation above has shown. But the remarkable finding here is that, under a general rotation, the different $\Gamma^{(\alpha)}$ do not mix, but they are just multiplied by a phase factor; indeed, since the rotation (16) implies that $\gamma\left(\boldsymbol{X}_{1}\right) \rightarrow \gamma^{\prime}\left(\boldsymbol{X}_{1}\right)=\gamma\left(\boldsymbol{X}_{1}\right) \mathrm{e}^{-2 \mathrm{i} \zeta_{1}}$, and similarly for the other two points, we see from the final expression in Eqs. (20) and (21) that the transformed invariants read

$\left(\Gamma^{(0)}\right)^{\prime}=\exp \left(-2 \mathrm{i}\left[\zeta_{1}+\zeta_{2}+\zeta_{3}\right]\right) \Gamma^{(0)}=\lambda_{2}^{(0)} \Gamma^{(0)}$,

and $\left(\Gamma^{(\alpha)}\right)^{\prime}=\lambda_{2}^{(\alpha)} \Gamma^{(\alpha)}$,

where the $\lambda_{2}^{(\alpha)}$ have been defined in Eq. (17). The transformation (22) is indeed remarkable, though in hindsight not all that surprising. The fact that the $\Gamma^{(\alpha)}$ only transform amongst themselves justifies that they are called natural components of the three-point correlation function of the shear. Note in particular that Eq. (22) implies that the four absolute values $\left|\Gamma^{(\alpha)}\right|$ are 
independent under general rotations; they are therefore the (non-linear) invariants of the shear 3PCF.

Of course, if desired, the original components of the shear 3PCF can be obtained from the natural components; the inverse of Eq. (21) reads:

$$
\begin{aligned}
& \gamma_{\mathrm{ttt}}=\left(\Gamma_{1}^{(1)}+\Gamma_{1}^{(2)}+\Gamma_{1}^{(3)}+\Gamma_{1}^{(0)}\right) / 4 ; \\
& \gamma_{\mathrm{t} \times \times}=\left(-\Gamma_{1}^{(1)}+\Gamma_{1}^{(2)}+\Gamma_{1}^{(3)}-\Gamma_{1}^{(0)}\right) / 4 ; \\
& \gamma_{\times \mathrm{t} \times}=\left(\Gamma_{1}^{(1)}-\Gamma_{1}^{(2)}+\Gamma_{1}^{(3)}-\Gamma_{1}^{(0)}\right) / 4 ; \\
& \gamma_{\times \times \mathrm{t}}=\left(\Gamma_{1}^{(1)}+\Gamma_{1}^{(2)}-\Gamma_{1}^{(3)}-\Gamma_{1}^{(0)}\right) / 4 \\
& \gamma_{\mathrm{tt} \times}=\left(\Gamma_{2}^{(1)}+\Gamma_{2}^{(2)}-\Gamma_{2}^{(3)}+\Gamma_{2}^{(0)}\right) / 4 \\
& \gamma_{\mathrm{t} \times \mathrm{t}}=\left(\Gamma_{2}^{(1)}-\Gamma_{2}^{(2)}+\Gamma_{2}^{(3)}+\Gamma_{2}^{(0)}\right) / 4 \\
& \gamma_{\times \mathrm{tt}}=\left(-\Gamma_{2}^{(1)}+\Gamma_{2}^{(2)}+\Gamma_{2}^{(3)}+\Gamma_{2}^{(0)}\right) / 4 \\
& \gamma_{\times \times \times}=\left(\Gamma_{2}^{(1)}+\Gamma_{2}^{(2)}+\Gamma_{2}^{(3)}-\Gamma_{2}^{(0)}\right) / 4
\end{aligned}
$$

\subsection{Parity transformation}

A parity transformation is obtained by mirroring a given set of three points with respect to any straight line. Such a transformation has the following effects: if in the original triangle the three points $\boldsymbol{X}_{1}, \boldsymbol{X}_{2}, \boldsymbol{X}_{3}$ are ordered counterclockwise with respect to, say, the center of the incircle, in the transformed triangle they will be ordered clockwise. As a result, under a parity transformation we need to replace every angle with its opposite.

In order to understand the behavior of the 3PCF under parity, let us suppose that the triangle is flipped along a line perpendicular to the side $\boldsymbol{x}_{3}$ (since the $3 \mathrm{PCF}$ is invariant upon rotation, the direction used for the flipping is irrelevant). We first observe that this mirror symmetry is equivalent to interchanging the points $\boldsymbol{X}_{1}$ and $\boldsymbol{X}_{2}$, or the sides $x_{1}$ and $x_{2}$. Second, such a flipping of orientation keeps the tangential component of the shear invariant (if the direction relative to which the shear components are measured are as well subject to the mirror transformation - like it happens when shear components are defined relative to one of the centers of the triangle), but changes the sign of the cross-component; hence, this transformation implies $P \gamma=\gamma^{*}$. Together, these two effects therefore imply that under a parity transformation,

$P\left[\gamma_{\mu \nu \lambda}\left(x_{1}, x_{2}, x_{3}\right)\right]=\Pi \gamma_{v \mu \lambda}\left(x_{2}, x_{1}, x_{3}\right)$,

where $\Pi=(-1)^{v+\mu+\lambda+1}$,

the parity, is +1 if all of the indices of $\gamma$ are t's, or two $\times$ 's occur, otherwise it is negative. Components of the $3 \mathrm{PCF}$ with $\Pi=+1$ are called even, those of negative parity odd components. The action of the parity transformation is most easily expressed in terms of the natural components of the $3 \mathrm{PCF}$; as can be seen from Eq. (21), the real part of each of the $\Gamma^{(\alpha)}$ is composed of even components, the imaginary part of odd components. Therefore,

$$
\begin{aligned}
P\left[\Gamma^{(0)}\left(x_{1}, x_{2}, x_{3}\right)\right] & =\left(\Gamma^{(0)}\right)^{*}\left(x_{1}, x_{3}, x_{2}\right)=\left(\Gamma^{(0)}\right)^{*}\left(x_{2}, x_{1}, x_{3}\right) \\
& =\left(\Gamma^{(0)}\right)^{*}\left(x_{3}, x_{2}, x_{1}\right), \\
P\left[\Gamma^{(1)}\left(x_{1}, x_{2}, x_{3}\right)\right] & =\left(\Gamma^{(1)}\right)^{*}\left(x_{1}, x_{3}, x_{2}\right)=\left(\Gamma^{(2)}\right)^{*}\left(x_{2}, x_{1}, x_{3}\right) \\
& =\left(\Gamma^{(3)}\right)^{*}\left(x_{3}, x_{2}, x_{1}\right), \\
P\left[\Gamma^{(2)}\left(x_{1}, x_{2}, x_{3}\right)\right] & =\left(\Gamma^{(1)}\right)^{*}\left(x_{2}, x_{1}, x_{3}\right)=\left(\Gamma^{(2)}\right)^{*}\left(x_{3}, x_{2}, x_{1}\right) \\
& =\left(\Gamma^{(3)}\right)^{*}\left(x_{1}, x_{3}, x_{2}\right), \\
P\left[\Gamma^{(3)}\left(x_{1}, x_{2}, x_{3}\right)\right] & =\left(\Gamma^{(1)}\right)^{*}\left(x_{3}, x_{2}, x_{1}\right)=\left(\Gamma^{(2)}\right)^{*}\left(x_{1}, x_{3}, x_{2}\right) \\
& =\left(\Gamma^{(3)}\right)^{*}\left(x_{2}, x_{1}, x_{3}\right) .
\end{aligned}
$$

Each of these parity transformations contains a permutation of the arguments with negative signature. One can also consider cyclic permutations of the arguments; since

$\gamma_{\mu \nu \lambda}\left(x_{1}, x_{2}, x_{3}\right)=\gamma_{\nu \lambda \mu}\left(x_{2}, x_{3}, x_{1}\right)=\gamma_{\lambda \mu \nu}\left(x_{3}, x_{1}, x_{2}\right)$,

one obtains for the natural components

$\Gamma^{(0)}\left(x_{1}, x_{2}, x_{3}\right)=\Gamma^{(0)}\left(x_{2}, x_{3}, x_{1}\right)=\Gamma^{(0)}\left(x_{3}, x_{1}, x_{2}\right)$,

$\Gamma^{(1)}\left(x_{1}, x_{2}, x_{3}\right)=\Gamma^{(3)}\left(x_{2}, x_{3}, x_{1}\right)=\Gamma^{(2)}\left(x_{3}, x_{1}, x_{2}\right)$,

$\Gamma^{(2)}\left(x_{1}, x_{2}, x_{3}\right)=\Gamma^{(1)}\left(x_{2}, x_{3}, x_{1}\right)=\Gamma^{(3)}\left(x_{3}, x_{1}, x_{2}\right)$,

$\Gamma^{(3)}\left(x_{1}, x_{2}, x_{3}\right)=\Gamma^{(2)}\left(x_{2}, x_{3}, x_{1}\right)=\Gamma^{(1)}\left(x_{3}, x_{1}, x_{2}\right)$.

Hence, $\Gamma^{(0)}$ is invariant under cyclic permutations of the arguments, whereas the other three natural components of the shear 3PCF transform into each other under such permutations. The relations (27) imply that only one of the three functions $\Gamma^{(k)}\left(x_{1}, x_{2}, x_{3}\right), 1 \leq k \leq 3$, is independent, the other two can be obtained by cyclic permutations of the arguments.

The parity transformations have an immediate consequence for triangles where two sides are equal, say $x_{1}=x_{2}$; namely, from Eq. (24) one finds that

$\gamma_{\mathrm{tt} \times}\left(x_{1}, x_{1}, x_{3}\right)=0=\gamma_{\times \times \times}\left(x_{1}, x_{1}, x_{3}\right)$;

$\gamma_{\mathrm{t} \times \mathrm{t}}\left(x_{1}, x_{1}, x_{3}\right)=-\gamma_{\times \mathrm{tt}}\left(x_{1}, x_{1}, x_{3}\right)$.

This implies that for $x_{1}=x_{2}, \Gamma^{(0)}$ and $\Gamma^{(3)}$ have no imaginary part, and those of $\Gamma^{(1)}$ and $\Gamma^{(2)}$ have equal magnitude but opposite sign. Furthermore, for equilateral triangles, all odd components of the shear 3PCF vanish, in which case the natural components become purely real.

\subsection{Two-point correlation function revisited}

The foregoing formalism can of course also be applied to the two-point correlation function. In that case, the eigenvalues are $\lambda_{1,2}^{(0,1)}=\mathrm{e}^{ \pm 2 \mathrm{i}\left(\zeta_{1} \pm \zeta_{2}\right)}$, and the invariant combinations are $\Gamma^{(0)}=\gamma_{\mathrm{tt}}-\gamma_{\mathrm{xx}}+\mathrm{i}\left(\gamma_{\mathrm{tx}}+\gamma_{\times \mathrm{t}}\right)$ and $\Gamma^{(1)}=\gamma_{\mathrm{tt}}+\gamma_{\mathrm{xx}}+\mathrm{i}\left(\gamma_{\mathrm{tx}}-\gamma_{\mathrm{xt}}\right)$. The two-point correlator is, however, special in the sense that after a parity transformation, the two points can be brought back into the old positions with a rotation. This then implies that the imaginary components of the $\Gamma^{(\alpha)}$ vanish if they are measured in the only reference frame that makes sense for two points - namely, the line connecting them; and so $\Gamma^{(0)}=\xi_{-}$, $\Gamma^{(1)}=\xi_{+}$. Note that if different projection directions are taken, the imaginary parts of the $\Gamma^{(\alpha)}$ are not zero. 


\subsection{Generalization}

The discussion on obtaining the natural components of the shear 3PCF immediately suggests how to generalize it to the natural components of higher-order correlation functions. Here, we shall give the results for the four-point function (also see the recent work by Takada \& Jain 2002a for a detailed consideration of the kurtosis of the cosmic shear field); further generalizations are straightforward to obtain:

If $\gamma_{\mu \nu \lambda k}$ is the four-point correlation function of the shear (for notational simplicity, we skip the arguments of this function), and we consider a general rotation of the directions relative to which tangential and cross components are defined, the transformation of the shear four-point correlation function reads

$$
\begin{aligned}
\gamma_{\mu \nu \lambda \kappa}^{\prime} & \equiv\left\langle\gamma_{\mu}^{\prime}\left(\boldsymbol{X}_{1}\right) \gamma_{v}^{\prime}\left(\boldsymbol{X}_{2}\right) \gamma_{\lambda}^{\prime}\left(\boldsymbol{X}_{3}\right) \gamma_{\kappa}^{\prime}\left(\boldsymbol{X}_{4}\right)\right\rangle \\
& =R_{\mu \alpha}\left(2 \zeta_{1}\right) R_{\nu \beta}\left(2 \zeta_{2}\right) R_{\lambda \gamma}\left(2 \zeta_{3}\right) R_{\kappa \delta}\left(2 \zeta_{4}\right) \gamma_{\alpha \beta \gamma \delta}
\end{aligned}
$$

In analogy to the treatment for the 3PCF, there are now eight complex conjugate pairs of eigenvalues, which are

$\lambda_{1,2}^{(0)}=\exp \left( \pm 2 \mathrm{i}\left[\zeta_{1}+\zeta_{2}+\zeta_{3}+\zeta_{4}\right]\right) ;$

$\lambda_{1,2}^{(1)}=\exp \left( \pm 2 \mathrm{i}\left[\zeta_{1}+\zeta_{2}-\zeta_{3}-\zeta_{4}\right]\right)$;

$\lambda_{1,2}^{(2)}=\exp \left( \pm 2 \mathrm{i}\left[\zeta_{1}-\zeta_{2}+\zeta_{3}-\zeta_{4}\right]\right)$;

$\lambda_{1,2}^{(3)}=\exp \left( \pm 2 \mathrm{i}\left[-\zeta_{1}+\zeta_{2}+\zeta_{3}-\zeta_{4}\right]\right)$;

$\lambda_{1,2}^{(4)}=\exp \left( \pm 2 \mathrm{i}\left[\zeta_{1}+\zeta_{2}+\zeta_{3}-\zeta_{4}\right]\right) ;$

$\lambda_{1,2}^{(5)}=\exp \left( \pm 2 \mathrm{i}\left[\zeta_{1}+\zeta_{2}-\zeta_{3}+\zeta_{4}\right]\right) ;$

$\lambda_{1,2}^{(6)}=\exp \left( \pm 2 \mathrm{i}\left[\zeta_{1}-\zeta_{2}+\zeta_{3}+\zeta_{4}\right]\right)$;

$\lambda_{1,2}^{(7)}=\exp \left( \pm 2 \mathrm{i}\left[-\zeta_{1}+\zeta_{2}+\zeta_{3}+\zeta_{4}\right]\right)$.

The corresponding natural components are then

$\Gamma^{(0)}=\left\langle\gamma\left(\boldsymbol{X}_{1}\right) \gamma\left(\boldsymbol{X}_{2}\right) \gamma\left(\boldsymbol{X}_{3}\right) \gamma\left(\boldsymbol{X}_{4}\right)\right\rangle ;$

$\Gamma^{(1)}=\left\langle\gamma\left(\boldsymbol{X}_{1}\right) \gamma\left(\boldsymbol{X}_{2}\right) \gamma^{*}\left(\boldsymbol{X}_{3}\right) \gamma^{*}\left(\boldsymbol{X}_{4}\right)\right\rangle$;

$\Gamma^{(2)}=\left\langle\gamma\left(\boldsymbol{X}_{1}\right) \gamma^{*}\left(\boldsymbol{X}_{2}\right) \gamma\left(\boldsymbol{X}_{3}\right) \gamma^{*}\left(\boldsymbol{X}_{4}\right)\right\rangle$;

$\Gamma^{(3)}=\left\langle\gamma^{*}\left(\boldsymbol{X}_{1}\right) \gamma\left(\boldsymbol{X}_{2}\right) \gamma\left(\boldsymbol{X}_{3}\right) \gamma^{*}\left(\boldsymbol{X}_{4}\right)\right\rangle$;

$\Gamma^{(4)}=\left\langle\gamma\left(\boldsymbol{X}_{1}\right) \gamma\left(\boldsymbol{X}_{2}\right) \gamma\left(\boldsymbol{X}_{3}\right) \gamma^{*}\left(\boldsymbol{X}_{4}\right)\right\rangle ;$

$\Gamma^{(5)}=\left\langle\gamma\left(\boldsymbol{X}_{1}\right) \gamma\left(\boldsymbol{X}_{2}\right) \gamma^{*}\left(\boldsymbol{X}_{3}\right) \gamma\left(\boldsymbol{X}_{4}\right)\right\rangle ;$

$\Gamma^{(6)}=\left\langle\gamma\left(\boldsymbol{X}_{1}\right) \gamma^{*}\left(\boldsymbol{X}_{2}\right) \gamma\left(\boldsymbol{X}_{3}\right) \gamma\left(\boldsymbol{X}_{4}\right)\right\rangle$;

$\Gamma^{(7)}=\left\langle\gamma^{*}\left(\boldsymbol{X}_{1}\right) \gamma\left(\boldsymbol{X}_{2}\right) \gamma\left(\boldsymbol{X}_{3}\right) \gamma\left(\boldsymbol{X}_{4}\right)\right\rangle$.

For reference, we shall write down the first of these explicitly,

$$
\begin{aligned}
\Gamma^{(0)}= & \gamma_{\mathrm{tttt}}+\gamma_{\times \times \times \times}-\gamma_{\mathrm{tt} \times \times}-\gamma_{\mathrm{t} \times \mathrm{t} \times}-\gamma_{\mathrm{t} \times \times \mathrm{t}}-\gamma_{\times \mathrm{tt} \times}-\gamma_{\times \mathrm{t} \times \mathrm{t}}-\gamma_{\times \times \mathrm{tt}} \\
& +\mathrm{i}\left[\gamma_{\mathrm{tt} \times \times}+\gamma_{\mathrm{tt} \times \mathrm{t}}+\gamma_{\mathrm{t} \times \mathrm{tt}}+\gamma_{\times \mathrm{ttt}}-\gamma_{\mathrm{t} \times \times \times}-\gamma_{\times \mathrm{t} \times \times}-\gamma_{\times \times \mathrm{t} \times}-\gamma_{\times \times \times \mathrm{t}}\right] .
\end{aligned}
$$

These natural components of the shear four-point correlation function will transform under the rotation (29) like

$\left(\Gamma^{(\alpha)}\right)^{\prime}=\lambda_{2}^{(\alpha)} \Gamma^{(\alpha)}$

These relations make it obvious how generalizations to higherorder correlations can be obtained.

\subsection{The estimator of Bernardeau et al. (2002)}

In their paper, Bernardeau et al. (2002b) considered, for the first time, a specific shear 3PCF, which they then applied successfully to observational cosmic shear data (Bernardeau et al. 2002a). Here, we shall write the Bernardeau et al. (2002b) estimator in our notation. They considered one side of a triangle (say, $\boldsymbol{x}_{3}$ ) as the reference direction, and project the shear at all three points along this direction. Hence, their projected components (here written as $\left.\gamma_{t, x}^{(3)}\right)$ read

$\gamma_{\mu}^{(3)}\left(\boldsymbol{X}_{l}\right)=-R_{\mu v}\left(2 \varphi_{3}\right) \gamma_{\nu}\left(\boldsymbol{X}_{l}\right)=R_{\mu \nu}\left(2 \varphi_{3}-2 \varphi_{l}\right) \gamma_{v}^{(\mathrm{s})}\left(\boldsymbol{X}_{l}\right)$,

and, of course, $\gamma^{(3)}\left(\boldsymbol{X}_{3}\right)=\gamma^{(\mathrm{s})}\left(\boldsymbol{X}_{3}\right)$. The 3PCF defined by Bernardeau et al. (2002b) then becomes

$\Gamma_{\mu}^{(\mathrm{B})}=\left\langle\left[\gamma_{\mathrm{t}}^{(3)}\left(\boldsymbol{X}_{1}\right) \gamma_{\mathrm{t}}^{(3)}\left(\boldsymbol{X}_{2}\right)+\gamma_{\times}^{(3)}\left(\boldsymbol{X}_{1}\right) \gamma_{\times}^{(3)}\left(\boldsymbol{X}_{2}\right)\right] \gamma_{\mu}^{(3)}\left(\boldsymbol{X}_{3}\right)\right\rangle$.

Since

$$
\begin{aligned}
\gamma_{\mathrm{t}}^{(3)}\left(\boldsymbol{X}_{1}\right) & \gamma_{\mathrm{t}}^{(3)}\left(\boldsymbol{X}_{2}\right)+\gamma_{\times}^{(3)}\left(\boldsymbol{X}_{1}\right) \gamma_{\times}^{(3)}\left(\boldsymbol{X}_{2}\right) \\
& =\left[R_{1 v}\left(2 \varphi_{3}-2 \varphi_{1}\right) R_{1 \lambda}\left(2 \varphi_{3}-2 \varphi_{2}\right)\right. \\
& \left.+R_{2 v}\left(2 \varphi_{3}-2 \varphi_{1}\right) R_{2 \lambda}\left(2 \varphi_{3}-2 \varphi_{2}\right)\right] \gamma_{v}^{(\mathrm{s})}\left(\boldsymbol{X}_{1}\right) \gamma_{\lambda}^{(\mathrm{s})}\left(\boldsymbol{X}_{2}\right) \\
& =R_{v \lambda}\left(2 \varphi_{1}-2 \varphi_{2}\right) \gamma_{v}^{(\mathrm{s})}\left(\boldsymbol{X}_{1}\right) \gamma_{\lambda}^{(\mathrm{s})}\left(\boldsymbol{X}_{2}\right),
\end{aligned}
$$

one obtains

$\Gamma_{\mu}^{(\mathrm{B})}=R_{v \lambda}\left(2 \phi_{3}\right) \gamma_{\nu \lambda \mu}^{(\mathrm{s})}$,

provided the triangle formed by the points $\boldsymbol{X}_{l}$ has the orientation defined at the beginning of Sect. 3 .

\section{Discussion and outlook}

The natural components of the shear 3PCF provide a generalization of the corresponding natural components $\xi_{ \pm}$of the two-point correlation function. Given that upcoming cosmic shear surveys will be substantially larger than the current ones, it is obvious that the 3PCF will be measurable in the future with a similar accuracy as the two-point correlation function in current surveys; in fact, a first significant measurement of the 3PCF has been reported in Bernardeau et al. (2002a). Therefore, it is worth to explore the dependence of the $3 \mathrm{PCF}$ on various parameters, as has been done for the two-point function. In particular, the detailed study of the interrelations between $\xi_{ \pm}$and the underlying power spectrum, as presented in Crittenden et al. (2002) and Schneider et al. (2002a), shall be generalized to the 3PCF. Of course, this will be substantially more difficult from a technical point of view, and will be deferred to future work. Nevertheless, we can outline a few aspects of what can be expected from such work.

In close analogy to the two-point correlation function, one can expect that the natural components can be more easily calculated from the bispectrum of the underlying mass distribution than the individual components of the 3PCF. As is the case for $\xi_{ \pm}$, one can expect that $\Gamma^{(0)}$ probes the bispectrum in a different way than the $\Gamma^{(k)}, 1 \leq k \leq 3$. In particular, the different natural components will have a different dependence on cosmological parameters. 
The natural components of the 3PCF are not independent of each other; provided that the shear indeed is due to a surface mass density field, there should be integral relations which interrelate them. Again one should note the analogy with the twopoint correlation function, where $\xi_{+}$can be obtained as an integral over $\xi_{\text {- }}$ and vice versa (Crittenden et al. 2002; Schneider et al. 2002a). The interrelations between the components of the 3PCF provide a redundancy which can be profitably combined to reduce the noise in real measurements.

If the shear is not solely due to a surface mass distribution, the shear field may contain a B-mode contribution (e.g., from intrinsic alignments of the galaxies from which the shear is measured). In the case of the two-point correlation function, the presence of a B-mode can be probed from integral relations between the two correlation functions $\xi_{ \pm}$(Crittenden et al. 2002; Schneider et al. 2002a); it is expected that similarly in the case of the 3PCF, the integral relations between the natural components will be modified in the presence of a B-mode.

All linear three-point statistics of the shear can be expressed in terms of the shear 3PCF. This is obvious from the fact that the bispectrum of the surface mass density can be expressed in terms of the 3PCF; on the other hand, all linear three-point statistics are linearly related to the bispectrum, and can therefore be expressed directly in terms of the 3PCF. In particular, the third-order aperture mass statistics (Schneider et al. 1998; van Waerbeke et al. 1999) can be expressed as an integral over the 3PCF. It remains to be seen whether the third-order aperture mass statistics is as useful for a separation of the shear field into E- and B-modes as it is the case for the two-point statistics (Crittenden et al. 2002; Schneider et al. 2002a; for applications in cosmic shear surveys, see e.g. Pen et al. 2002; Hoekstra et al. 2002).

Concerning practical measurements of the 3PCF, the procedure is straightforward: from a given catalog of galaxy images with position vectors $\boldsymbol{\theta}_{i}$ and measured ellipticities $\epsilon_{i}$, triplets are selected. For each such triplet, the sides of the corresponding triangle can be calculated, and for practical reasons the largest side be called $x_{3}$. The three points are then labeled $\boldsymbol{X}_{l}, 1 \leq l \leq 3$ in the (unique) way such that the orientation of the points is as described at the beginning of Sect. 3, and the longest side connects $\boldsymbol{X}_{1}$ and $\boldsymbol{X}_{2}$. Since the connecting vectors $\boldsymbol{x}_{l}$ need to be calculated anyway, it is simplest to project the ellipticities along the sides of the triangle, or, with opposite sign, towards the orthocenter, without having to calculate any trigonometric function. The eight $(=2 \times 2 \times 2)$ triple products of the projected ellipticities are calculated and summed up in eight threedimensional bins. Those are conveniently labeled by a scale $x_{3}$, and two shape parameters, $q_{1}=x_{1} / x_{3}, q_{2}=x_{2} / x_{3}$, so that the grid of bins runs as $0 \leq q_{1,2} \leq 1,0<x_{3}<\infty$. After summing up over all triplets of points, for each of the eight components the sums in the bins are divided by the number of triplets contributing, which then yields an estimate of the 3PCF. Those can then be combined into the natural components for further analysis.

For a quantitative analysis of the three-point statistics of cosmic shear in relation to predictions of cosmological models, one can either use integrated properties of the shear 3PCF such as the aperture mass or the integral of $\Gamma^{(\mathrm{B})}$ over an elliptical region as done in Bernardeau et al. (2002b), or consider the (more noisy, but much more numerous) estimates of the natural components of the 3PCF directly. The first method yields one-dimensional functions of the three-point shear statistics depending on a scale parameter, and are thus very convenient for graphical displays. In contrast, using the full shear 3PCF employs multi-dimensional data which is difficult to display, but contains all the information about thirdorder statistics from the data. Hence, even though the signal-tonoise in each bin of the 3PCF can be small, its overall information content cannot be smaller than that of any of the integrated quantities, and should therefore be employed in extracting cosmological information from the measurements. To obtain a reliable figure-of-merit for the comparison of the observational results with model predictions, one needs to know the covariance matrix of the shear 3PCF - this, however, is a 64-components quantity depending on 6 arguments, and will be very difficult to obtain analytically (see Schneider et al. 2002b for the difficulties of obtaining the covariance of the two-point correlation functions). Corresponding covariances of integrated quantities may be slightly easier obtainable. On the other hand, it is quite likely that figures-of-merit will have to rely heavily on future ray-tracing simulations through $N$-body-generated cosmological mass distributions, as in, e.g., van Waerbeke et al. (1999), Jain et al. (2000) and Bernardeau et al. (2002a).

After this paper was finished, two recent preprints were posted on the Web which discussed very similar issues (Zaldarriaga \& Scoccimarro 2002; Takada \& Jain 2002b). In the latter one, the components $\gamma_{\mu \nu \lambda}$ of the correlation function were calculated from ray-tracing simulations; in particular they verified that all of the eight components of the 3PCF are nonzero in general and thus contain cosmological information.

Acknowledgements. We are grateful to Matthias Bartelmann for very useful comments on this manuscript. This work was supported by the TMR Network "Gravitational Lensing: New Constraints on Cosmology and the Distribution of Dark Matter" of the EC under contract No. ERBFMRX-CT97-0172, by the German Ministry for Science and Education (BMBF) through the DLR under the project 50 OR 0106, and the Deutsche Forschungsgemeinschaft under the project SCHN 342/3-1.

\section{References}

Bacon, D. J., Refregier, A. R., \& Ellis, R. S. 2000, MNRAS, 318, 625 Bacon, D., Massey, R., Refregier, A., \& Ellis, R. 2002 [astro-ph/0203134]

Bartelmann, M., \& Schneider, P. 1999, A\&A, 345, 17

Bartelmann M., Schneider P., 2001, Phys. Rep., 340, 291

Bernardeau, F., van Waerbeke, L., \& Mellier, Y. 1997, A\&A, 322, 1

Bernardeau, F., Mellier, Y., \& van Waerbeke, L. 2002a, A\&A, 389, L28

Bernardeau, F., van Waerbeke, L., \& Mellier, Y. 2002b [astro-ph/0201029]

Blandford, R. D., Saust, A. B., Brainerd, T. G., \& Villumsen, J. V. 1991, MNRAS, 251, 600

Crittenden, R. G., Natarajan, P., Pen, U.-L., \& Theuns, T. 2002, ApJ, 568,20

Hämmerle, H., Miralles, J.-M., Schneider, P., et al. 2002, A\&A, 385, 743 
Hamana, T., Colombi, S. T., Thion, A., et al. 2002, MNRAS, 330, 455

Hoekstra, H., Yee, H., \& Gladders, M. 2002 [astro-ph/0204295]

Jain, B., \& Seljak, U. 1997, ApJ, 484, 560

Jain, B., Seljak, U., \& White, S. D. M. 2000, ApJ, 530, 547

Kaiser, N. 1992, ApJ, 388, 272

Kaiser, N. 1998, ApJ, 498, 26

Kaiser, N., Wilson, G., \& Luppino, G. 2000 [astro-ph/0003338]

Maoli, R., van Waerbeke, L., Mellier, Y., et al. 2001, A\&A, 368, 766

Mellier, Y. 1999, ARA\&A, 37, 127

Miralda-Escudé, J. 1991, ApJ, 380, 1

Pen, U.-L., van Waerbeke, L., \& Mellier, Y. 2002, ApJ, 567, 31

Refregier, A., Rhodes, J., \& Groth, E. J. 2002 [astro-ph/0203131]

Rhodes, J., Refregier, A., \& Groth, E. J. 2001, ApJ, 552, L85

Schneider, P. 1996, MNRAS, 283, 837

Schneider, P., van Waerbeke, L., Jain, B., \& Kruse, G. 1998, MNRAS, 296,873
Schneider, P., van Waerbeke, L., \& Mellier, Y. 2002a, A\&A, 389, 729

Schneider, P., van Waerbeke, L., Kilbinger, M., \& Mellier, Y. 2002b, A\&A, 396, 1

Takada, M., \& Jain, B. 2002a [astro-ph/0205055]

Takada, M., \& Jain, B. 2002b [astro-ph/0210261]

van Waerbeke, L., Bernardeau, F., \& Mellier, Y. 1999, A\&A, 243, 15

van Waerbeke, L., Mellier, Y., Erben, T., et al. 2000, A\&A, 358, 30

van Waerbeke, L., Mellier, Y., Radovich, M., et al. 2001, A\&A, 374, 757

van Waerbeke, L., Mellier, Y., Pello, R., et al. 2002 [astro-ph/0202503]

White, M., \& Hu, W. 2000, ApJ, 537, 1

Wittman, D. M., Tyson, J. A., Kirkman, D., Dell'Antonio, I., \& Bernstein, G. 2000, Nature, 405, 143 